\title{
Formularies, costs, and quality of care
}

\section{Formulary restrictions are not the answer, especially for epilepsy}

David M. Labiner, MD

Kendra W. Drake, MD

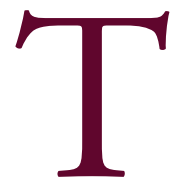

he goal of treating an individual with epilepsy is to have no seizures and no side effects. Limiting availability of medications appears to be a simple way of controlling costs of patient care. This approach potentially jeopardizes both efficacy and safety. We argue, in this edition of Current Controversies, that limiting costs by restricting formularies is detrimental to the patients from an efficacy, safety, and cost perspective.

It is well known that the cost of medical care is soaring. In an attempt to keep costs of care under control, many have advocated limiting formularies. The idea is to decrease the direct cost of care to individuals by limiting the availability of expensive medications, especially when there are more cost-effective alternatives. While

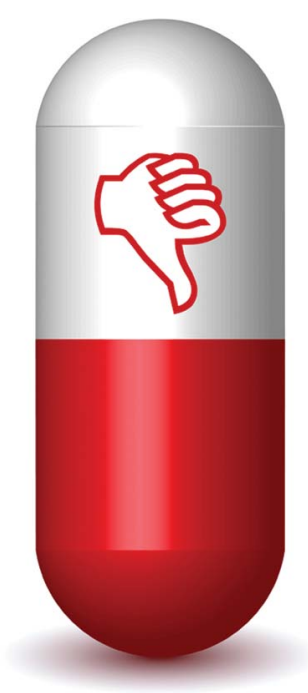
this may make some sense in certain disease states, we would argue that epilepsy is different and as such, formularies must include access to all antiepileptic drugs (AEDs), both generic and branded, in order to improve both seizure control and quality of life.

Unlike hypertension or hyperglycemia, seizures (and by extension epilepsy) are an allor-none phenomenon. Unlike a small fluctuation in blood pressure or glucose levels, a single seizure can have significant psychosocial and physical consequences, including injury, limitations in driving or employment, hospitalization, and sudden unexpected death, to name a few. For this reason, formularies must have many effective and safe options available to help render patients free of both seizures and side effects.

How then should we establish which AEDs should be included in formularies? First and foremost, it should be demonstrated that the medications are effective and safe. There is little evidence to suggest that available AEDs fail to meet these criteria. While there is no doubt that most individuals will respond favorably to the first medication they try, this is not a universal truth, with over $30 \%$ with refractory seizures despite appropriate therapy. ${ }^{1}$ In fact, many individuals will require switching to a different medication, or combination of medications,

Departments of Neurology (DML, KWD) and Pharmacy Practice \& Science (DML), Colleges of Medicine and Pharmacy, The University of Arizona, Tucson.

Correspondence to: labinerd@neurology.arizona.edu 


\section{While there is no doubt that most individuals} will respond favorably to the first medication they try, this is not a universal truth, with over $30 \%$ with refractory seizures despite appropriate therapy.

due to either lack of efficacy or intolerable side effects. An unrestricted formulary providing numerous options for rational therapy and polytherapy, with novel mechanisms of action and side effect profiles, makes this possible. A strategy that has been implemented for availability of HIV or Parkinson medications, with multiple medications with differing mechanisms of action, makes sense for AEDs as well. This approach assures availability of the widest variety of medications to manage these chronic illnesses and to obtain seizure freedom as quickly as possible given the potential significant consequences of refractory seizures.

There is little direct comparative efficacy data available by which to compare older, cheaper AEDs with their newer, more expensive brethren. There are, however, evidence-based reviews that provide scientific data to support the thesis that the newer medications are effective both as monotherapy and add-on therapy in newly diagnosed as well as treatment-resistant epilepsy. ${ }^{2,3}$ Perhaps the more important observation in these reports is that the side effect profile of the newer medications appears better than those seen with older AEDs. This was also reported, but downplayed, in the recent controversial AHRQ review of AEDs. ${ }^{4}$ It is difficult to argue that decreases in cognitive side effects, teratogenesis, depression, and damage to bone health are somehow less desirable. This is in fact the case with the newer AEDs as a group when compared to the older AEDs. Additionally, the newer medications, in aggregate, have fewer interactions with other concomitant medications than the older AEDs. One can rightly argue that these are reviews that are not head-to-head comparisons of the medications in question. It is also true that carbamazepine has become the gold standard for treating localizationrelated seizures and that no antiseizure medication has been shown to be more effective. While this position is no doubt true, a large multicenter study comparing carbamazepine with two newer medications demonstrated equal efficacy but fewer side effects associated with the newer medications. ${ }^{4}$

The same data cited above ${ }^{1}$ show that over one-third of patients with epilepsy will likely never respond favorably to AEDs. While this is a strong argument for pursuing nonpharmacologic treatments, it also supports the need for large formularies for treating seizures as epilepsy is a heterogeneous disorder for which a single medication or a single mechanism of action would not be effective in all cases. There is no disputing the observation that there is a diminishing return with the trial of each new AED in a given individual. It has been observed in nearly all randomized controlled trials of new AEDs that the seizure reduction is modest and the seizure freedom rate is low. However, the response is not nil. Rather, in each of these studies, in patients with treatment-resistant epilepsy (medically refractory), there is a seizure-free rate of up to $5 \%$. Further, roughly half of the patients will be clinical responders, those with greater than $50 \%$ reduction in their seizure frequency. While this is without a doubt underwhelming to some, it is extremely meaningful to the individuals whose seizures diminish or to those who become seizure-free.

Formulary restrictions can present in other ways that are potentially detrimental to individuals with epilepsy. These include "fail-first" models where an individual must typically use a cheaper alternative and fail (have seizures) prior to gaining access to the complete formulary. Since a single seizure can be detrimental (see above) or even cause death, this is a risky policy. 


\section{There is a growing body of evidence (without adequate well-designed trials to directly address the question) that formulation substitution of AEDs can lead to increased emergency treatment, injury, hospitalizations, and hence cost.}

The other limiting approach is to require mandatory generic substitution, which is also complicated by the formulary's ability to substitute one brand of generic for another without notifying either the patient or physician. There is a growing body of evidence (without adequate well-designed trials to directly address the question) that formulation substitution of AEDs can lead to increased emergency treatment, injury, hospitalizations, and hence cost. ${ }^{5}$ This should not be construed as an argument against the use of low-cost generic medications in general but rather a caution that their use without sensible controls may be "pennywise but pound foolish." One such control would be the use of single source of a product so that the formulation substitutions that may underlie the findings in these studies would be eliminated.

In our minds, the only argument that can be made to limit the availability of antiepileptic medications from formularies is one of cost. Others can attempt to make arguments regarding the comparative effectiveness of AEDs. In fact, this claim might be valid if such data existed. However, the limited, and flawed, data we have suggest that the cheaper, older medications have more ill effects than the newer medications. The ability of the treating physician to properly select the most appropriate medication for the optimal treatment of an individual with epilepsy or other seizure disorder while taking into account the individual's comorbidities, age, past experiences, and medication interactions requires the availability of a wide variety of medications.

Although there are no specific data to show that formulary restriction has produced worse outcomes, we would echo but alter a recommendation (regarding generics) from an editorial in Lancet Neurology, "Until firm evidence supporting the restriction of formularies becomes available, we should err on the side of caution and ensure that AEDs are excluded from any sweeping policies that promote such limitation."

\section{REFERENCES}

1. Kwan P, Brodie MJ. Early identification of refractory epilepsy. N Engl J Med 2000;345:314-319.

2. Glauser T, Ben-Menachem E, Bourgeois B, et al. ILAE treatment guidelines: evidence-based analysis of antiepileptic drug efficacy and effectiveness as initial monotherapy for epileptic seizures and syndromes. Epilepsia 2006;47:1094-1120.

3. French JA, Kanner AM, Bautista J, et al. Efficacy and tolerability of the new antiepileptic drugs II: treatment of refractory epilepsy. Neurology 2004;62:1261-1273.

4. Talati R, Scholle JM, Phung OJ, et al. Effectiveness and Safety of Antiepiltpic Medications in Patients with Epilepsy: Comparative Effectiveness Review No. 40. AHRQ Publication No. 11(12)-EHC082EF. Rockville, MD: Agency for Healthcare Research and Quality; 2011. Available at: www.effectivehealthcare.ahrq.gov/reports/final.cfm.

5. Labiner DM, Paradis PE, Duh MS, et al. Generic antiepileptic drugs and associated medical resource utilization in the United States. Neurology 2010;74:1566-1574.

\section{STUDY FUNDING}

No targeted funding reported. 


\section{DISCLOSURES}

D.M. Labiner receives grant funding, including salary support, from the Centers for Disease Control and Prevention. He receives research funding, without salary support, from Eisai, Sepracor, SK Life Science, Upsher-Smith Labs, and Visualase. K.W. Drake receives grant funding, including salary support, from the Centers for Disease Control and Prevention. She is on the speakers' bureau for UCB Pharma. Go to Neurology.org/cp for full disclosures.

\section{Get the Latest Drug Recalls and Warnings. Give the} Best Patient Care

The American Academy of Neurology and the Health Care Notification Network have teamed up to offer AAN members a FREE service that delivers timely neurology-specific FDA-mandated patient safety drug alerts directly to your e-mail inbox.

Don't miss this opportunity to provide the best-and safest-possible care for your patients: visit www.aan.com/view/FDAalerts. 